\title{
Application of Stratified Training on Physical Education Training
}

\author{
Haoqin Li \\ Zhengzhou University of Industrial Technology, Zhengzhou, Henan, 451150
}

Keywords: Physical Education, Stratified Training, Application Study

\begin{abstract}
Physical education is a kind of culture, while physical education is a kind of cultural transmission. With the teaching content and teaching facilities being determined, with the new concept of curriculum reform being infiltrated into PE teaching, the research and reform of PE teaching methods play an important role in implementing the $\mathrm{PE}$ curriculum reform. The application of hierarchical teaching in physical education is a bold attempt.
\end{abstract}

\section{Introduction}

In the past, the teaching methods of physical education generally used the teacher to verbally describe the teaching routine of adding students 'imitation practice. The choice of teaching content did not necessarily meet the requirements of the current students' physical exercise. The teaching methods were not designed to stimulate the passion of students. As a result, Physical education classroom completely devoid of vitality and vitality. Therefore, the use of stratified sub-teaching method in PE teaching in colleges and universities will be able to make up for this teaching blank. Guided by the multi-level teaching thought according to local conditions and based on the actual development needs of students, we design personalized teaching methods for students by designing targeted teaching methods, which not only enrich the content of classroom teaching, but also improve students' training Effect is a great role in promoting the use of stratified teaching method in the teaching of physical education in higher vocational education for the improvement of the teaching atmosphere has played a positive role in promoting.

\section{Sports stratified sub-teaching method advantages}

The current education reform has repeatedly emphasized the importance of all-round development of students, according to the actual needs of students physical training for students tailored to their development of physical education methods. Under the guidance of the Unified Physical Education Syllabus, students in different grades, students of different genders, students with different physical qualities and students with different physical training ability should be guided by local conditions so that each student can learn something. For example, in regular training, a physical education class is not only to carry out long-distance sports as the main content, but through a comprehensive assessment of physical strength, endurance for its designated training subjects targeted for teachers to endurance has reached the standard Of students develop appropriate physical training subjects, such as horizontal bars, parallel bars, rings and so on, so that students can effectively use their energy to improve their physical quality [1].

In the past, the teaching mode was not only the one-size-fits-all teaching content but also the consistent uniform standard of academic testing, rather than testing the actual learning effect. For example, students were given the only sprint running test items. Students with different physical conditions Is obviously unfair, and some students like running, good at running, then the examination subjects for the test of the actual physical ability of the students simply fail to detect the role, and for students who are not usually good at such projects, and adopted Uniform standard of compliance will obviously make this part of the students seem powerless, even though the sprint run through normal training a little improvement in the project, but in the absolute evaluation criteria, there may be no performance at all, no doubt the training of students Enterprising cause a great blow. The teaching method of stratification and sub-division requires that examination items 
should be formulated according to the students 'height, weight, physical exercise experience, physical condition and other comprehensive problems so that the examination of students' training effects fully embodies humanization [2].

Although the state has made great efforts to increase the support for schools in colleges and universities, it is difficult to realize the equitable provision of physical education for each student in light of the large base of our country as a whole, the large number of students, the large amount of funds allocated to the school's hardware resources, Training equipment requirements, therefore, in the actual teaching of physical education to solve this problem should start from the teaching methods, the hierarchical sub-teaching methods effectively into the clues for the solution to the current problems. For example, the number of shots in schools is obviously inadequate to meet the requirements of all students for unified shot training. Teachers can teach students according to their hobbies and interests so that part of the students can perform shot training while others have strut Training, two different ways of exercise not only meet the purpose of students' physical training, but also help to optimize the allocation of physical education resources. In addition, they also appropriately reflect the guiding ideology of teaching students in accordance with their aptitudes and according to local conditions.

\section{Layered training teaching design}

The choice of teaching content should pay attention to the adaptability of students 'physical development and psychological development. The students in higher vocational education are in the critical period of physical growth. The students' bones, body shape, internal organs and muscles also possess some degree of plasticity. Student psychology is more sensitive, easy to come from outsiders evaluation and opinion ideas, therefore, in the arrangement of physical education content in addition to pay attention to quantitative principles, but also pay attention to the principle of qualitative. For example, during the daily morning maneuvers, there should be more control over the alignment of maneuvers and the proportional coordination of boys and girls. Students physical exercise is not the sole purpose of physical education. In the process of physical exercise, the cultivation of students 'spiritual life is also an embodiment of effective teaching of physical education. In physical education, teachers should pay attention to stepping up the guidance of the students' correct ideological and moral development direction to infiltrate students to cultivate the sense of social honor and sense of social responsibility, in sports activities should actively advocate the development of students mutual aid and love, unity and cooperation [3].

For example, in the long jump training, teachers should pay attention to strengthening the interaction and cooperation among students so as to enable students to set up training mutual aid groups on their own so as to enable students with insignificant training effects to carry out useful physical training under the leadership of sports health leaders. Students can guide the students with a little poorer performance in various aspects such as preparation for long jump, long-jump run, long-jump take-off, long-jump precautions and so on. Students can not only improve their physical training skills but also expand physical education through communication between students and students Range.

Colleges and universities are the bases for training specialized talents. As the name suggests, specialized personnel are those who possess the skills of a skill. Therefore, the specialty is undoubtedly the characteristic of teaching in colleges and universities. The teaching of specialization should also be appropriately reflected in the teaching of physical education in colleges and universities. Teachers 'teaching design should be aimed at students' different majors. The study of different professional skills requires the cooperation of different body languages of the students and the long-term use of the same body. It is likely to cause partial discomfort to the body. Therefore, physical education should be combined with the use of different organs of the body To provide students with the purpose of physical health. For example, students majoring in computer, language and accounting may easily become nervous due to sedentary and prolonged mental work. Therefore, it is appropriate to include sports activities that are conducive to general relaxation such as volleyball, soccer, Gymnastics and so on. For civil engineering, nursing, numerical control and 
other professional students, due to the need for a long time to stand, will increase the pressure on body organs, over time caused by poor blood circulation, leg congestion, varicose veins and other issues, therefore, in physical education should be appropriate To join the sports activities that enhance coordination between different organs of the body, such as tennis, drawbar, swimming and table tennis. For marketing, tourism, construction, automotive and other professional students, due to the need for a long period of high frequency walk, unconsciously will increase the degree of physical fatigue, psychology at home to bear some pressure, it is easy to students of cardiovascular and cerebrovascular Therefore, in physical education, endurance endurance training should be properly adopted, such as martial arts, swimming, playing ball and so on [4].

First, the principle varies from person to person, according to the actual situation of students to select the teaching content of science, setting teaching methods, and setting teaching goals, to achieve targeted, developmental teaching design, so as to achieve the purpose of enhancing the effectiveness of student training, which is the core idea ofthe hierarchical teaching method. In the teaching of physical education in higher vocational schools, teachers should flexibly adjust their teaching plans according to the difficulty of teaching tasks so as to make them suitable for the development and progress of each student. They should not avoid the students' Training is too easy to ignore the occurrence of teaching activities. In the choice of teaching tasks, teachers should attach importance to the overall situation of the students, to develop training methods of different levels and different projects for the purpose of unified teaching so that students can get the same training effect through different training ways.

Second, the principle of gradual and orderly progress, as sports can be trained and studied in other cultural subjects, follow the principle of gradual and orderly progress. Physical fitness training does not take place overnight. Teachers and students need long-term friendly cooperation to achieve the desired results. In the arrangement of PE teaching activities, teachers should adhere to the principle of appropriate amount. One motor skill learning can not be based on specific chapters or class hours. Instead, it should formulate a development strategy based on the actual development of students. All in order to benefit students' mind and body Healthy development as the center. The implementation of the principle of gradual and orderly should adhere to the simple to complex, from the general to the special process of evolution, so that the body is constantly familiar with and adapt to certain motor functions, and gradually form the motor function. Teachers should implement a dynamic management strategy for students' motivation so that students can make clear the next development task under the condition of satisfactorily completing the current tasks, and teachers should pay attention to the connection and connection between training tasks at different levels to enable students to have the physical training Zhang relaxation of the transition. For example, when teaching students' basketball integrated sports skills, teachers should adopt the teaching methods of breaking through one by one. First, students should be taught to practice different basketball skills such as dribbling, passing and shooting. After students master all basic basketball skills On the basis of only organized students to carry out the entire series of basketball comprehensive exercise to enable students to master the skill without having to exercise due to the system can not coordinate and organize movement agitation [5].

In the research process, we summarize the following simple, easy to operate several evaluation methods:

Focus on individual differences. This method is an assessment of a student object itself as a reference point.

Scientific use of absolute evaluation method. That is, the target of teaching as an objective reference point, in the evaluation, the object of evaluation and objective criteria for comparison, evaluation of each student's compliance level. In operation, different levels of students to adopt different objective criteria to enable students to maintain normal psychological state.

Relative evaluation method. Relative evaluation method refers to a collective (class), with the collective average score as a reference point, evaluate each academic position in the collective.

Incentive evaluation method. This evaluation method is to capture students in the learning activities of the flash point and the success of the guide, in order to meet the best psychological 
needs of students to mobilize their innovative enthusiasm.

\section{Conclusions}

The reform of PE teaching activities in tertiary institutions is a concrete manifestation of current education and teaching reform. It is a manifestation of realizing the combination of students 'learning ability and physical quality development. It is a manifestation of realizing the all-round development of students' The effective use of methods can not only effectively enhance the students' ability of physical training, but also benefit the cultivation of students' solidarity and cooperation, which is of great help to promote the students' all-round development.

\section{References}

[1] Wang Haidong. Amateur sports training of secondary school students re-understanding [J]. Inner Mongolia Sports Science and Technology. 2005 (03):16-18.

[2] The right to talk about the psychological health of secondary vocational students participating in sports training [J]. Contemporary Sport Science and Technology. 2017 (21):152-155.

[3] Ma Weijun. Study on the Use of Scenario Games in High School Physical Education Training [J]. Journal of Asia-Pacific Education. 2016 (25):213-214

[4] Yang Xiaohui enrich physical education classroom activities, to strengthen the physical training of secondary vocational students [J] life of 2017. (23):67-69.

[5] Huang Lili. On sports training theory and method [J]. Tomorrow fashion 2016 (13):82-83. 